\title{
Multi-Sensor Chip for Monitoring Key Parameters in Bioprocesses
}

\author{
Nurul Izni Rusli, Irene Pia Vincentini, Frederik Ceyssens, and Michael Kraft
}

\begin{abstract}
This paper presents a feasibility study on the integration of multiple biosensors based on amperometric, potentiometric, and impedimetric detection techniques, as well as physical sensors on a single chip. The sensor chip comprises six sensors for in-situ monitoring of important process parameters in a bioprocess: glucose, lactate, $\mathrm{pH}$, cell density, dissolved oxygen concentration, and temperature. The waferlevel parallel fabrication process of multiple microsensors on a single substrate involves four relatively simple processes. The fabricated enzyme sensors were tested with a linear working range up to a concentration of $10 \mathrm{mM}$ for the glucose and lactate sensors and show a fast response time of $20 \mathrm{~s}$. The integrated pH sensor was governed by a near-Nernstian equation with a sensitivity of $60.8 \pm 2.4 \mathrm{mV} / \mathrm{pH}$. Cell density, dissolved oxygen, and temperature sensor were also electrically tested. Results obtained from experimental measurements can be considered a promising step towards the realization of a real-time and parallel in-situ measurement platform for bioprocesses.
\end{abstract}

Index Terms-Amperometric, bioprocesses monitoring, cell density, dissolved oxygen, electrochemical and biological sensors, glucose, impedimetric, integrated multi-sensor, $\mathrm{pH}$, potentiometric, temperature.

\section{Introduction}

$\mathrm{P}$ RECISE control of multiple parameters influencing bioprocesses is crucial to meet the increasing requirements in production and research. These parameters include concentration of nutrients (e.g. glucose) and metabolites (e.g. lactate), $\mathrm{pH}$, cell density, oxygen level, and temperature [1], [2]. To monitor these key parameters, numerous analytic methods have been employed [2]-[6]. Among the desirable attributes of monitoring methods is the ability to monitor parameters in real-time, which has been commonly used for parameters such as $\mathrm{pH}$, oxygen level, and temperature [5], [7][9]. However, real-time measurements are considerably more difficult for monitoring nutrients, metabolites, and cellular components.

N. I. Rusli thanks Ministry of Higher Education Malaysia (MOHE) and Universiti Malaysia Perlis (UniMAP) for the financial support under sponsorship Skim Latihan Akademik Bumiputera (SLAB) Reference No. KPT(BS)860703295192.

N. I. Rusli is with the Department of Electrical Engineering (ESATMNS), University of Leuven (KU Leuven), 3001 Leuven, Belgium, on study leave from the Department of Electrical Engineering Technology, Faculty of Engineering Technology, Universiti Malaysia Perlis (UniMAP), 02100 Padang Besar, Perlis, Malaysia (e-mail: nurulizni.rusli@esat.kuleuven.be).

I. P. Vincentini was with the DEIB, Politecnico di Milano, 20133 Milano, Italy (e-mail: irenepia.vincentini@mail.polimi.it).

F. Ceyssens is with the Department of Electrical Engineering (ESATMICAS), University of Leuven (KU Leuven), 3001 Leuven, Belgium (e-mail: fceyssen@esat.kuleuven.be).

M. Kraft is with the Department of Electrical Engineering (ESATMNS), University of Leuven (KU Leuven), 3001 Leuven, Belgium (e-mail: michael.kraft@kuleuven.be)
Spectroscopic methods such as ultraviolet-visible [10], nearinfrared [11], [12], mid-infrared [13], Raman, and fluorescence [14] may appear as promising techniques to monitor multiple analytes in real-time. The use of spectroscopy, however, often suffers from low selectivity, and requires knowledge on chemometrics and multivariate data analysis [3], [5], [15]. Accordingly, this technology has been only slowly adopted by industry, which may also be due to the investment cost required to implement new instrumentation or the higher confidence of process engineers in established conventional electrochemical sensors [3]. Thus, there is still a need for reliable, low cost, smaller size, and continuous sensor systems with greater selectivity.

Advancements in microfabrication techniques have enabled miniaturization and new multi-parametric electrochemical (bio)sensors devices. The integration of miniaturized and multiple sensors on a single substrate would be desirable for many applications that require high performance, small form factor, low power consumption, and low cost. For example, Internet of Things (IoT) applications often require numerous sensor devices that respond to a variety of chemical, biological, and physical parameters. These tiny sensors need to be interfaced with wireless power transmission system and sensing readout circuitry. At present, most sensors have their own specific fabrication process, interface circuit, package, test, and calibration methodology. In the field of portable and mobile platforms, this approach of using a separate individual sensor for measuring each parameter of interest is no longer suitable and outdated. This is due to considerations of many factors such as size, power consumption, and the economic aspect. The trend 
is shifting towards multi-sensor platforms that incorporate multiple sensing elements for wearable electronics, health, environmental, food, and process monitoring as well as home automation sensor systems [16], [17].

Multi-parameter sensor platforms based on electrochemical transduction would offer a promising solution to simultaneously monitor a wide range of parameters owing to their smaller size, cheaper cost, good compatibility, rapid response, and ability to provide sufficient sensitivity for detection of analytes as well as physical quantities [1], [3]. Using such platforms, continuous real-time monitoring of analytes in bioprocess may also become feasible with in-situ measurement, requiring no physical sampling. This real-time method is highly desirable because of various advantages such as detecting small, local changes rapidly, adjusting relevant parameters, and maintaining the process at optimum condition [1]. Although such platforms have been demonstrated for bioprocesses monitoring [1], [7], [18]-[20], research on the subject has only been carried out for a small number of sensing modalities; no single work demonstrating the integration of six important process parameters on a single chip exists.

In contrast, in this study the integration of six electrochemical biosensors based on amperometric, potentiometric, impedimetric/conductometric detection techniques, as well as physical sensors on a single chip has been demonstrated. The rationale for this development is that it would miniaturize the measurement setup, reduce device footprint as well cost, and hence makes the device more commercially viable for application in a wireless, IoT-style sensor node. The paper describes the design and microfabrication process as well as a demonstration of the functionality and operability of all six integrated sensors. Detailed experimental data analyses are shown in the results section.

\section{Sensor Design And InTEgRAted Process}

\section{A. Enzyme Sensors}

Enzyme-based amperometric biosensing has been adopted in this work due to its simplicity, high selectivity, and sensitivity in complex environments [21]. In the design of enzyme sensors (i.e. glucose and lactate), a three-electrode configuration with a working electrode (WE), counter electrode (CE), and reference electrode (RE) was adopted. These electrodes were arranged in concentric circles to ensure even distribution of the current density between the WE and $\mathrm{CE}$, and independence of the direction flow in stirred solution [22]. Furthermore, this circular shape makes it easier to dispense the enzyme solution onto the WE area (see Fig. S3 in the Supplementary Information).

The design and size of the electrodes was chosen based on previous work of Mross et al. [23], [24], in which the droplet formation that dispensed by microliter pipettes was studied. The footprint of this circular sensing structure was $\sim 1.57 \mathrm{~mm}^{2}$, with a WE area of $\sim 0.26 \mathrm{~mm}^{2}$.

Amperometry is an electrochemical technique of analysis in which the potential applied to the measuring cell is kept constant while the response current depends on the concentration of the electroactive particles in the cell. The operation of these amperometric biosensors is based on selective enzymatic conversion of glucose or lactate molecules (in presence of oxygen - a cosubstrate of the enzymatic reaction) by an enzyme which results in generation of electrochemically active hydrogen peroxide $\left(\mathrm{H}_{2} \mathrm{O}_{2}\right)$, subsequent oxidation of the generated $\mathrm{H}_{2} \mathrm{O}_{2}$ yields two electrons at positive potential, and detection of these electrons can be registered as an oxidation current proportional to substrate concentration by a potentiostat [1], [22], [23]. The corresponding reactions for the enzymes glucose oxidase and lactate oxidase are given in (1) and (2), respectively. The $\mathrm{H}_{2} \mathrm{O}_{2}$ which was oxidized at the WE yields two electrons per molecule is given in (3).

$$
\begin{aligned}
& \mathrm{B} \text {-D-glucose }+\mathrm{O}_{2} \rightarrow \text { D-gluconic acid }+\mathrm{H}_{2} \mathrm{O}_{2} \\
& \text { L-lactate }+\mathrm{O}_{2} \rightarrow \text { pyruvate }+\mathrm{H}_{2} \mathrm{O}_{2} \\
& \mathrm{H}_{2} \mathrm{O}_{2} \rightarrow \mathrm{O}_{2}+2 \mathrm{H}^{+}+2 e^{-}
\end{aligned}
$$

\section{B. $\mathrm{pH}$ Sensor}

The designed two-electrode parallel-plate potentiometric $\mathrm{pH}$ sensor utilizes a polymer-based ion-selective WE to detect hydrogen ions $\left(\mathrm{H}^{+}\right)$. The RE was made of silver $(\mathrm{Ag})$ with silver chloride $(\mathrm{AgCl})$ layer, while the WE was made of gold $(\mathrm{Au})$ as conductive electrode material because of good results that have been achieved in the previous work [25]. In the potentiometry technique, the concentration of the analyte of interest in a solution is quantified by measuring the potential between a $\mathrm{pH}$ sensitive WE and RE as a result of the free energy change due to chemical interactions, occurring between the sensing materials and $\mathrm{H}^{+}$[26]. This potential difference is taken as a measure for quantitatively determining the $\mathrm{H}^{+}$concentration in solution by the Nernst equation, and consequently the $\mathrm{pH}$ [27].

Polymer-based potentiometric $\mathrm{pH}$ electrodes are opted in our multi-sensor design because they are biocompatible, easy to fabricate, small-footprint, stable, and cheap. Commonly used $\mathrm{H}^{+}$selective polymers are polypyrolle, polyaniline (PANI), and poly(1-aminoanthracene) [26]-[28]. In this work, the Au WE surface was modified with PANI as this has been commonly used in the previous works for $\mathrm{pH}$ sensing owing to its reversible protonation/deprotonation reaction of emeraldine salt-emeraldine base transition (acid-base reaction) and the reported high stability, low cost, straightforward preparation procedure [26], [28], [29]. Among several techniques available to prepare conducting polymer films, electrochemical in-situ polymerization is preferred as it allows better control of film coverage and thickness on the specified active electrode surface [30]. We have designed several variants for the $\mathrm{pH}$ sensors but the presented data in this work are for the WE area of $0.25,0.30$, 0.40 , and $0.48 \mathrm{~mm}^{2}$.

\section{Cell Density Sensor}

The cell density sensor based on impedance spectroscopy measurements was designed with an interdigitated electrodes (IDEs) configuration. The two-IDEs impedance-based cell density sensor measures the impedance of a cell suspension below and above the characteristic frequency, $f_{\text {char }}$ of the cells. The impedance below $f_{\text {char }}$ is a function of the viable cell mass whereas the upper frequency impedance is not influenced by the cells. This method is in general able to differentiate between live and dead cells, because dead cells without intact membranes do not contribute to charge polarization [2], [31]. The polarization occurs up to a certain frequency, which is known as the characteristic frequency $f_{\text {char }}$ for a certain cell type. When the $f_{\text {char }}$ is exceeded, the charges at the cell membrane 
cannot follow the electric field and do not contribute to the measured impedance anymore. The cell density can be derived from the measured impedance spectrum as it is related to the conductivity of the cell suspension below $f_{\text {char }}$ [32]. As been reported by Yang et al. [33], impedance of cell suspension in a certain frequency range is related to the cell concentration in the suspension and thus, different cell concentrations can result in different electrical impedance spectral responses. A typical impedance spectrum in aqueous solution exhibit two cut-off frequencies with an ohmic plateau in between. This ohmic plateau is a representative of the solution resistance, $R_{\text {sol }}$ and this value could be taken as a measure for the cell density. The IDEs were designed to have an impedance spectrum with an ohmic plateau around $f_{\text {char }}$ of yeast cells, i.e. $\sim 5 \mathrm{MHz}$. Based on the previous work reported by Mross et al. [1], [23], the lower and upper cut-off frequencies of the impedance spectrum in phosphate buffered saline (PBS) were estimated as a function of number of interdigitated finger $N$, finger length $L$, width $W$, and spacing $S$. Several variants with different parameters were designed but the data discussed in this work are for IDEs with $N=43, L=1000 \mu \mathrm{m}, W=37 \mu \mathrm{m}$, and $S=20 \mu \mathrm{m}$.

\section{Dissolved Oxygen Sensor}

A three-electrode configuration was adopted for our dissolved oxygen $(\mathrm{dO})$ sensor. The electrodes consist of a platinum (Pt) $\mathrm{WE}$ and $\mathrm{CE}$, as well as an $\mathrm{Ag} / \mathrm{AgCl} \mathrm{RE}$. The sensor operation is based on an amperometry technique, adopting a microelectrode array (MEA) as WE for reliable, smaller electrode sizes to reduce oxygen consumption and accurate detection of oxygen [34]. The reaction mechanism is based on electrochemical reduction of oxygen at the WE, given in (4).

$$
\mathrm{O}_{2}+2 \mathrm{H}^{+}+2 e^{-} \rightarrow \mathrm{H}_{2} \mathrm{O}_{2}
$$

The MEA were fabricated using $\mathrm{Pt}$ conductors and a Parylene-C insulating layer on the glass substrate. Parylene-C has been chosen as insulating material due to its process compatibility with existing microelectronics fabrication technologies, making it suitable for low-cost applications. The results that are presented in this work are for a parallel-plate $\mathrm{dO}$ sensor consisting of a square $\mathrm{CE}$, a square RE (both being $500 \mu \mathrm{m} \times 500 \mu \mathrm{m}$ in size), and an MEA-based WE (25 MEs; $8 \mu \mathrm{m} \varnothing ; 80 \mu \mathrm{m}$ spacing).

\section{E. Resistance Temperature Device}

In order to reduce device footprint, a meander-line thin-film resistance temperature device (RTD) was designed. The RTD is based on the measured resistivity of a conductive metal as temperature changes. With rising temperature the movement of charge carriers in the metal is increasingly impeded, hence increasing the resistance of metallic layer. The relationship between resistance $R$ and temperature $T$ can be expressed by polynomial equation and its simplified version as given in (5) and (6), respectively [35]:

$$
\begin{aligned}
& R_{\mathrm{T}}=R_{0}\left[1+A\left(T-T_{0}\right)+B\left(T-T_{0}\right)^{2}+C\left(T-T_{0}\right)^{3}+\ldots\right] \\
& R_{\mathrm{T}}=R_{0}\left[1+A\left(T-T_{0}\right)\right]
\end{aligned}
$$

where $R_{0}$ is resistance at the reference temperature $T_{0}$ (usually either $0{ }^{\circ} \mathrm{C}, 20^{\circ} \mathrm{C}$, or $25^{\circ} \mathrm{C}$ ); $A, B, C, \ldots$ are material-dependent temperature coefficients (in ${ }^{\circ} \mathrm{C}^{-1},{ }^{\circ} \mathrm{C}^{-2},{ }^{\circ} \mathrm{C}^{-3}, \ldots$ ). Among the materials that most commonly used for RTDs, Pt is by far the most adopted material because of its long-term chemical stability, broad temperature range, near linear $R-T$ relationship, and high positive temperature coefficient of resistance of $3.9 \times 10^{-3} \mathrm{C}^{-1}$ [35]. Considering the fact that most of the sensors in our multi-sensor chip are based on Pt electrodes, the use of Pt as RTD material will simplify the fabrication process. Patterning of Parylene-C insulation layer on top of RTDs to provide mechanical and moisture protection for the RTDs also could be performed at wafer-level in parallel with the MEA fabrication for $\mathrm{dO}$ sensors. The $\mathrm{DC}$ value of $R$ can be estimated from the number of squares of metal in the structures, neglecting the skin effect [36]-[38]. Thus, considering the meander-line resistor configuration as shown in Fig. 1, the series resistance is given by (7):

$$
R=\rho \frac{L}{t w}=R_{\mathrm{sh}} \frac{h N+d(N-1)+2 b}{w}
$$

where $R_{\mathrm{sh}}=\rho / t$ is the sheet resistance metal layer used for the resistor design and $N$ is the number of line segments. In this work, the thickness $t$, and the length $b$ were fixed to $200 \mathrm{~nm}$ and $1.5 \mathrm{~mm}$ (at least), respectively. These values were taken as the ideal values of the deposited Pt layer height by neglecting the Titanium (Ti) adhesion layer $\sim 20 \mathrm{~nm}$ and also the nominal distance of chip active area from the chip edge, as discussed in $\mathrm{S} 1$ in Supplementary Information. Three different variants were calculated, designed, and fabricated considering different resistance at reference temperature of $20^{\circ} \mathrm{C}\left(R_{20}{ }^{\circ} \mathrm{C}\right)$ as shown in Table I by taking $\rho_{20}{ }^{\circ} \mathrm{C}=1.06 \times 10^{-7} \Omega \mathrm{m}$ as reference value [39], [40]. These test samples are designated as $\mathrm{Pt}_{20}{ }^{\circ} \mathrm{C} 100$, $\mathrm{Pt}_{20}{ }^{\circ} \mathrm{C} 500$, and $\mathrm{Pt}_{20}{ }^{\circ} \mathrm{C} 1000$ for $R_{20}{ }^{\circ} \mathrm{C}$ of $100 \Omega, 500 \Omega$, and $1000 \Omega$, respectively.

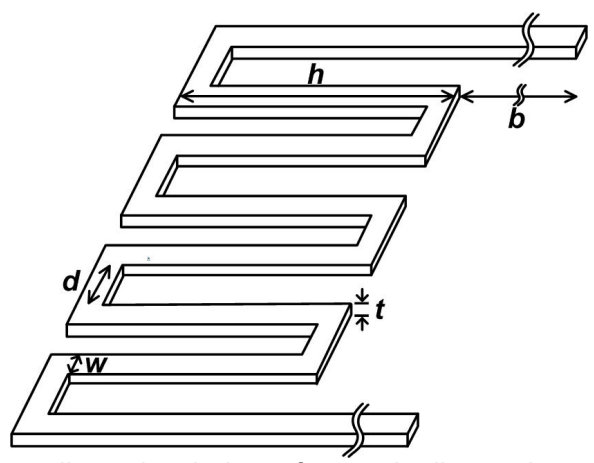

Fig. 1. Three-dimensional view of meander-line resistor with design parameters $b, w, d, t$, and $h$; number of lines $N$ is 8 (in this case).

TABLE I

DESIGN VARIANTS FOR RTDS

\begin{tabular}{llllllll}
\hline \hline Variant & $\begin{array}{l}\mathrm{R}_{20}{ }^{\circ} \mathrm{C} \\
(\Omega)\end{array}$ & $\begin{array}{l}b \\
(\mu \mathrm{m})\end{array}$ & $\begin{array}{l}w \\
(\mu \mathrm{m})\end{array}$ & $\begin{array}{l}d \\
(\mu \mathrm{m})\end{array}$ & $\begin{array}{l}h \\
(\mu \mathrm{m})\end{array}$ & $N$ & $\begin{array}{l}L \\
(\mu \mathrm{m})\end{array}$ \\
\hline $\mathrm{Pt}_{20}{ }^{\circ} \mathrm{C} 100$ & 100 & 1600 & 27 & 50 & 436 & 4 & 5094 \\
$\mathrm{Pt}_{20}{ }^{\circ} \mathrm{C} 500$ & 500 & 1700 & 10 & 50 & 710 & 8 & 9434 \\
$\mathrm{Pt}_{20}{ }^{\circ} \mathrm{C} 1000$ & 1000 & 1700 & 5 & 50 & 710 & 8 & 9434 \\
\hline \hline
\end{tabular}

\section{F. Integration Process}

The selection of the microfabricated sensors' design and configuration over other types of sensors and analytical methods are based on the following criteria; (1) easier integration with other sensors and better compatibility with the additive fabrication technology, (2) wider sensing range and faster response with comparable sensitivity, and (3) lower cost. 
Additional details on the sensor integration are described in S1 of Supplementary Information.

\section{Materials And Methods}

\section{A. Materials and Reagents}

S1818 photoresist and LOR10B lift-off resist were purchased from Micro Resist Technology (Berlin, Germany). AZ® 351B developer, N-Methyl-2-pyrrolidone (NMP) solvent, hexamethyldisilizane (HMDS) adhesion promoter, and TechniEtch A180 were purchased from MicroChemicals (Ulm, Germany). Borofloat 33 glass wafers was purchased from Drix NV. Iron (III) chloride $\left(\mathrm{FeCl}_{3}\right)$, potassium chloride $(\mathrm{KCl})$, Nafion $^{\text {TM }}$ perfluorinated resin solution, N,N'dimethylformamide, aniline, glucose oxidase (GOx), glutaraldehyde solution, bovine serum albumin (BSA), D-(+)glucose powder, sodium L-lactate, L-ascorbic acid, L-cysteine, ready-to-use Certipur ${ }^{\circledR}$ buffer solutions, and external RE were purchased from Sigma Aldrich (Overijse, Belgium). Hydrochloric acid $(\mathrm{HCl})$ were purchased from Fisher Chemical $^{\mathrm{TM}}$. Lactate oxidase (LOx) was purchased from A.G. Scientific (San Diego, California). Phosphate buffered saline (PBS) tablets was purchased from VWR.

\section{B. Fabrication Process}

Sensor prototypes were fabricated on a $7 \mathrm{~mm} \times 7 \mathrm{~mm}$ chip area, comprising six sensors for monitoring of important process parameters: glucose, lactate, $\mathrm{pH}$, cell density, $\mathrm{dO}$, and temperature as shown in Fig. 2. These sensors were realized using a four-mask process that allowed to fabricate $\mathrm{Pt}, \mathrm{Au}$, and $\mathrm{Ag}$ electrodes, and connections on a glass substrate as well as a patterned Parylene-C insulation layer. Thus, each metal layer serves multiple purposes across the different sensors, limiting fabrication complexity. Further details about the fabrication are included in S1 of the Supplementary Information. Techniques for wafer-level surface modifications for $\mathrm{AgCl} \mathrm{RE}$ and $\mathrm{pH}$ sensors are described in S2 of the Supplementary Information. Localized enzyme functionalization on top of WE of enzyme sensors (i.e. glucose and lactate sensors) are also added to S2. Details on the measurements that have been carried out are included in S3 of the Supplementary Information.

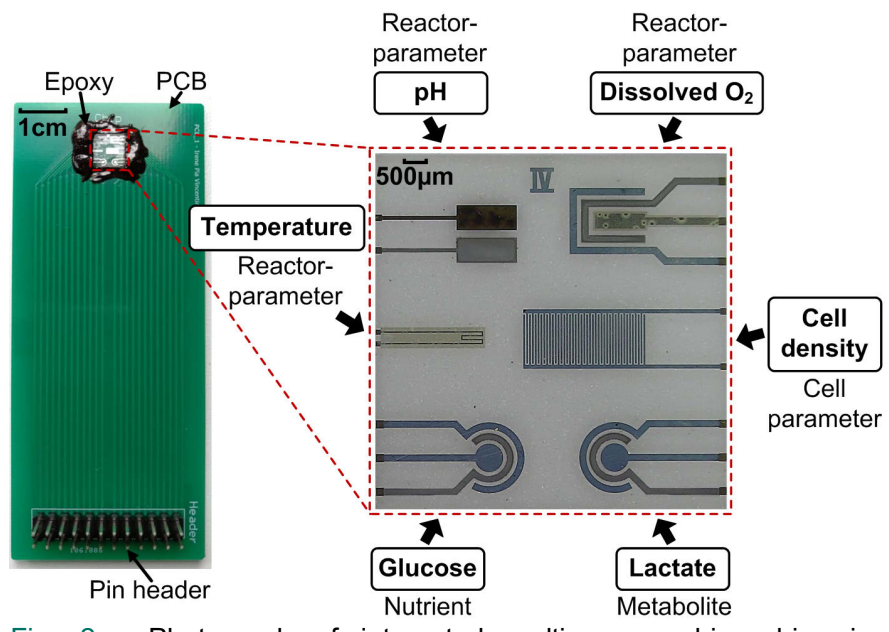

Fig. 2. Photograph of integrated multi-sensor chip; chip size $7 \mathrm{~mm} \times 7 \mathrm{~mm}$. The sensors were arranged in a way that allowed to place the bond pads on the left and right side of the chip, such that a dual in-line package could be used for future integration.

\section{RESULTS AND DISCUSSION}

\section{A. Enzyme Sensors}

To determine the applicability of the sensor, we measured the enzymatic sensor response for concentrations between $0.5 \mathrm{mM}$ and $60 \mathrm{mM}$ of glucose and lactate. Fig. 3 shows the chronoamperometric (CA) responses of the glucose sensor and lactate sensor to glucose and lactate, respectively. The current significantly increases with the stepwise increase of analyte concentration in a solution from 0.5 to $10 \mathrm{mM}$ for both sensors. However, as the analyte concentration was increased further, no significant increase in current was observed for both sensors. At higher substrate concentrations, the superposition of the ambient noise levels from using a magnetic stir plate and the noise associated with electrochemical reaction at the electrodeelectrolyte interface [41], were significantly amplified and visibly seen as current fluctuations especially for lactate sensor which has much lower current response as compared to glucose sensor. The response time was less than $20 \mathrm{~s}$ for both sensors. Both sensors react sufficiently fast for real-time measurements of glucose and lactate levels.

The graphs of sensor current as a function of glucose concentration in the inset of Fig. 3 show that the current increases linearly for concentrations between 0.5 to $10 \mathrm{mM}$ of glucose and lactate. The correlation coefficients which were determined by the least-squares method were found to be 0.9595 and 0.8966 for the glucose and lactate sensors, respectively. At higher concentration of glucose and lactate, the current responses were no more linearly proportional to the concentrations and became constant, likely due to the saturation of the enzymatic reaction. The sensor response for higher concentration of glucose and lactate in solution follows typical Michaelis-Menten kinetics control with the response being limited by lack of oxygen at the reaction site (stoichiometric restrictions) [42]. This oxygen deficiency results in saturation of the sensor for high glucose levels and limitation of the linear region. Our findings on the linear working region are slightly higher than those reported by Liu et al. [43] and Tatiana et al. [44], however almost 10-fold less than those reported by Sato et al. [45]. This was to be expected since sensors of varying geometry, surface area, and different functionalization and measurement protocols were utilized in the respective studies.

The standard deviation from the mean was below $5.5 \%$ and $21 \%$ for glucose and lactate sensor, respectively. This might be due to variations in the manual electrode functionalization of GOx and LOx (as described in S2 in the Supplementary Information), making the enzyme layer thickness less consistent, and thus affect the sensitivity of the sensors. The standard deviation of the lactate sensor is a little worse, exhibiting $21 \%$, which can be attributed to the lower current measured due to lower activity and poor stability of LOx than the GOx [44].

The linear measurement range of the sensors could be extended by additional diffusion membrane coatings as diffusion control to limit the transport of the analyte in relation to the oxygen transport on top of enzyme layer [46]. This additional entrapment method of enzyme immobilization could also possibly further improve the enzyme stability and leaching problem. In this case, the most common polymer used as selective membrane are polyurethane and Nafion. However, 
this application of an additional membrane would very often result in a trade-off between high signals or sensitivity and a linear range [21]. For instance, sensitivity of a lactate sensor would be sacrificed if the linear range needs to be extended.

We have also demonstrated investigations on enzyme-based sensors' selectivity and stability, as they are key parameters for creating effective analytical systems intended for operation with real samples. The selectivity and stability results are included in S4 and S5, respectively, in the Supplementary Information. These investigations will further guide optimization on surface modifications to improve the selectivity and stability, especially for the lactate sensor. Further investigations will focus on enhanced linear range measurement, selectivity, and the attainment of efficient interference rejection.

Nevertheless, this preliminary analysis demonstrated by the amperometric enzyme sensors (i.e. glucose and lactate sensor) proved its functionality, notwithstanding its significantly smaller sensing area of $\sim 0.26 \mathrm{~mm}^{2}$. The enzyme immobilization technique employed in this study also proved that the enzyme could be fixed to the electrode without major loss in activity to allow continuous and sensitive sensor performance.

\section{B. $p H$ Sensor}

The open-circuit potential responses with respect to external $\mathrm{Ag} / \mathrm{AgCl} \mathrm{RE}$ as a function of $\mathrm{pH}$ showed a decreasing potential relative to $\mathrm{pH}$, as also reported in previous work [27], [29], [47], [48]. Measurements on the fabricated prototype for PANImodified electrode samples were governed by a near-Nernstian behaviour with an average sensitivity of $60.8 \pm 2.4 \mathrm{mV} / \mathrm{pH}$ between $\mathrm{pH}$ of 4 and $\mathrm{pH} 10$ with a high linear regression coefficient, as illustrated in Fig. 4. It is worth noting that the measured average sensitivity differs only $2.8 \%$ from the theoretical value of Nernstian response, which is $59.16 \mathrm{mV} / \mathrm{pH}$ [27]. Similar results were indeed reported previously [27], [29], [47]. The bare electrode showed poor sensitivity with low regression coefficient. The relative standard deviation attributed to the measurements of $\mathrm{pH} 10$ for PANI electrode was found to be higher (exceed $10.5 \%$ ) than the measurements of $\mathrm{pH} 2$ and 4. It has been reported that PANI-based $\mathrm{pH}$ sensors exhibit strong deprotonation at $\mathrm{pH}>9$ which limits the use of PANI as potentiometric $\mathrm{pH}$ sensor to measurements between $\mathrm{pH} 2$ and 9 [47], [49]. After this proof-of-concept, with the $\mathrm{pH}$ sensor functionality proven, this $\mathrm{H}^{+}$ion-selective $\mathrm{pH}$ sensor based on PANI will be further characterized and optimized.

\section{Cell Density Sensor}

The Bode plot of impedance spectra for two-interdigitated electrodes (IDEs) cell density sensor in PBS solution are shown in Fig. 5. The curves reveal an ohmic plateau from approximately $150 \mathrm{kHz}$ to $10 \mathrm{MHz}$ which provides a suitable frequency range for cell density measurements. To further evaluate the influence of different yeast suspensions (changing the conductivity of the solution medium), the impedance responses were measured. When the yeast suspension increases in cell density, the $R_{\text {sol }}$ decreases (the plateau shifts to lower impedance) as also reported by Yang [33]. The plateau value depends on the quantity of polarizable cell membranes in the medium. Increasing the yeast suspension increases the cell number as well as the number of polarizable cell membrane, and consequently leads to an overall increase in conductivity.

\section{Dissolved Oxygen Sensor}

Fig. 6(a) shows the cyclic voltammetry (CV) curves for oxygen reduction under atmospheric conditions in PBS (ca. $67 \%, 5.85 \mathrm{mg} / \mathrm{l}$ ) and in oxygen-deprived conditions in PBS (ca. $25 \%, 2.18 \mathrm{mg} / \mathrm{l}$ ). As shown in the graph, the oxygen reduction takes place in a potential range between -0.2 and $-0.7 \mathrm{~V}$ vs. $\mathrm{Ag} / \mathrm{AgCl}$. Within this range, a potential of $-0.2 \mathrm{~V}$ is chosen for further CA characterization. Fig. 6(b) shows the resulting $\mathrm{CA}$ curves for different $\mathrm{dO}$ concentration in PBS. Higher absolute current values in CA curves correspond to larger $\mathrm{dO}$ concentrations. As can be observed in the inset of Fig. 6(b), the absolute averaged current detected in oxygen deprived PBS increases as the $\mathrm{dO}$ concentration increases, with good linear correlation $\left(\mathrm{R}^{2}=0.9978\right)$. A similar response trend was indeed reported previously [6], [50]. These findings validated the ability of the MEA dO sensor to measure oxygen level.

\section{E. Resistance Temperature Device}

Fig. 7 shows the measured electrical resistance over temperature for six RTDs test samples with 3 different variants, which have been measured 3 times for each sample. As the temperature was increased from $10^{\circ} \mathrm{C}$ to $70^{\circ} \mathrm{C}$, in steps of $5^{\circ} \mathrm{C}$, the resistance of all RTDs samples increased, exhibiting temperature dependence of electrical resistance in the deposited Pt. $\mathrm{Pt}_{20}{ }^{\circ} \mathrm{C} 1000$ samples showed the highest slope which was directly proportional to the resistance at $20^{\circ} \mathrm{C}, R_{20}{ }^{\circ} \mathrm{C}$. However, the relative standard deviations of the $\mathrm{Pt}_{20}{ }^{\circ} \mathrm{C} 1000$ samples are the highest, but never exceeded $0.50 \%$ for each sample. The sensitivity of RTDs over a given range of temperature is defined by its temperature coefficient resistance TCR $(\alpha)$, which can be calculated using (8) [51]:

$$
\alpha=\left(\frac{1}{R_{T_{i}}}\right) \frac{\left(R_{T_{i}}-R_{T_{f}}\right)}{\left(T_{i}-T_{f}\right)}
$$

where $R_{T 1}$ and $R_{T \mathrm{f}}$ are the resistance values obtained at initial temperature $T_{\mathrm{i}}$ and final temperature $T_{\mathrm{f}}$. The values of the ideal and measured $R_{20}{ }^{\circ} \mathrm{C}$, as well as calculated average $\alpha$ are summarized in Table II.

TABLE II

MeAsured AND CALCulated Values for RTDS

\begin{tabular}{llll}
\hline \hline Variant & $\begin{array}{l}\text { Ideal } \mathrm{R}_{20}{ }^{\circ} \mathrm{C} \\
(\Omega)\end{array}$ & $\begin{array}{l}\text { Measured Mean } \mathrm{R}_{20}{ }^{\circ} \mathrm{C} \\
(\Omega)\end{array}$ & $\begin{array}{l}T C R, \alpha \\
\left(\% /{ }^{\circ} \mathrm{C}\right)\end{array}$ \\
\hline $\mathrm{Pt}_{20}{ }^{\circ} \mathrm{C} 100$ & 100 & $227.7 \pm 5.5$ & 0.28 \\
$\mathrm{Pt}_{20}{ }^{\circ} \mathrm{C} 500$ & 500 & $1082.2 \pm 10.5$ & 0.26 \\
$\mathrm{Pt}_{20}{ }^{\circ} \mathrm{C} 1000$ & 1000 & $2092.8 \pm 23.6$ & 0.28 \\
\hline \hline
\end{tabular}

The TCR was calculated considering the resistances recorded at initial temperature of $10^{\circ} \mathrm{C}$ and final temperature of $70{ }^{\circ} \mathrm{C}$. The measured $R_{20}{ }^{\circ} \mathrm{C}$ values were at least 2-fold higher than the ideal $R_{20}{ }^{\circ} \mathrm{C}$ which were used in the theoretical calculation to determine the parameters for the meander-line structure. This deviation is most likely due to the higher resistivity of the sputtered Pt thin film due to the combination effects of fine grain sizes and electron confinement effects of the sputtered metal thin films [52], [53]. Recalculating the resistivity of the deposited Pt thin film using the measured design parameters of 

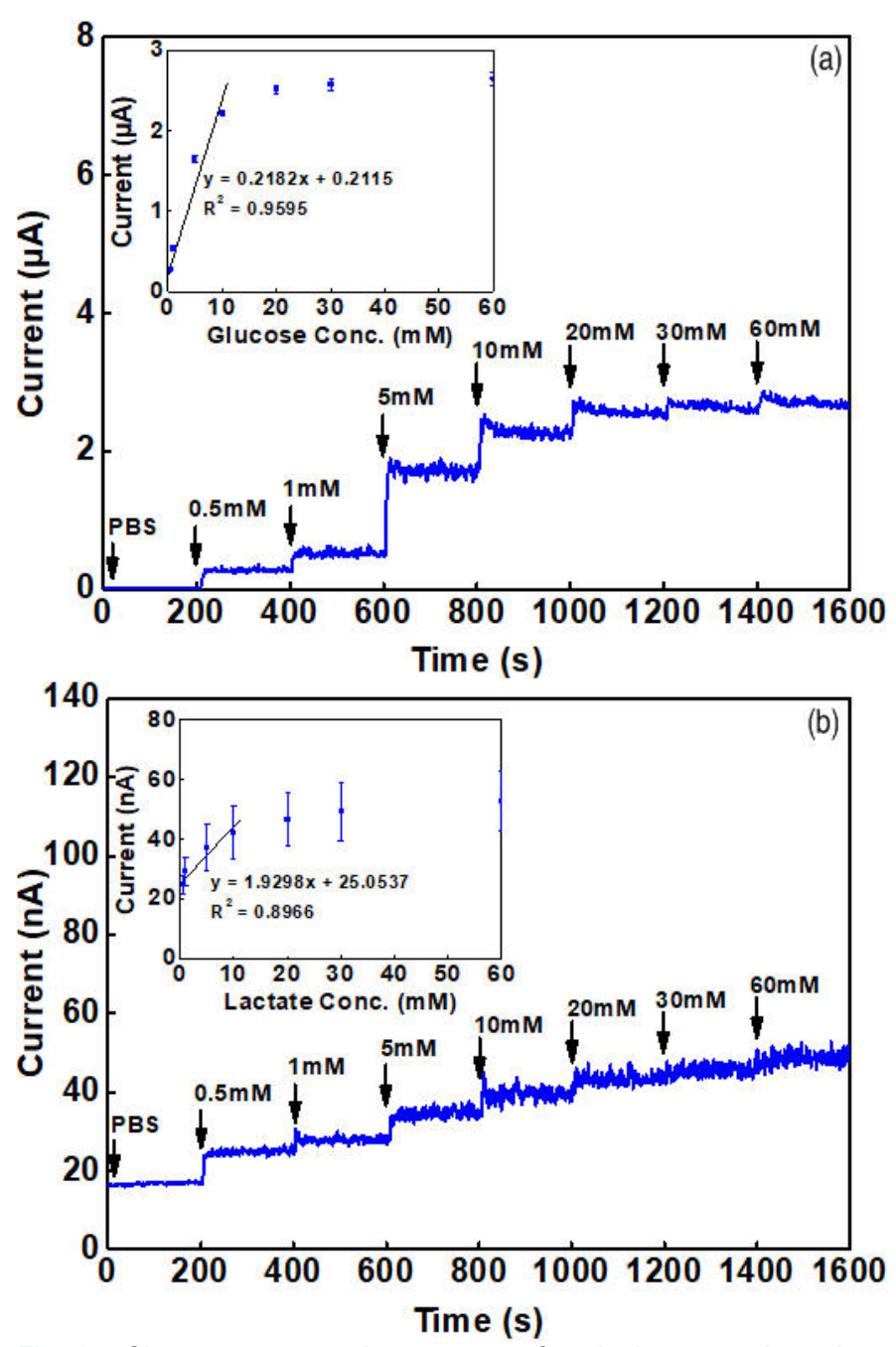

Fig. 3. Chronoamperometric response of a single sensor in various (a) glucose, and (b) lactate concentrations. Increasing substrates concentrations were added every $200 \mathrm{~s}$ and arrows in plots correspond to cumulative concentrations at different time intervals. The inset shows the measured sensor current as a function of (a) glucose, (b) lactate concentration and the data were averaged from measurements of 3 different sensors. The error bars represent the standard deviation.
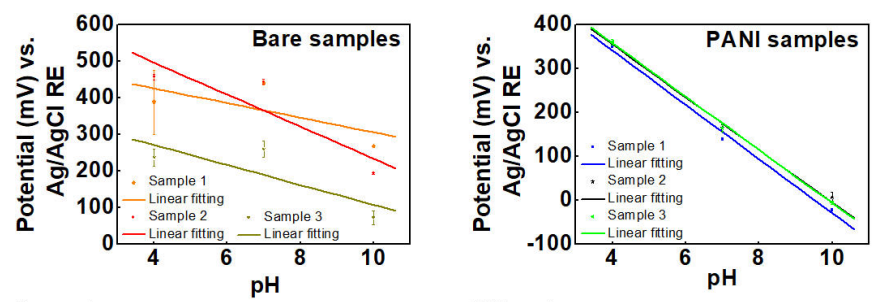

Bare samples

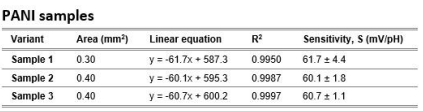

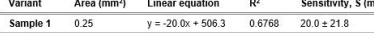
\begin{tabular}{lllll}
\hline Sample 2 & 0.40 & $y=-43.9 \times+572.7$ & 0.8871 & $43.9 \pm 22.9$ \\
\hline Sample 3 & 0.49 & $y=-27.4 \times+391.1$ & 0.0042 & $27.4 \pm 20.2$ \\
\hline
\end{tabular}

Fig. 4. Open-circuit voltage responses with respect to the external $\mathrm{Ag} / \mathrm{AgCl} \mathrm{RE}$ as a function of $\mathrm{pH}$ for three bare and three PANI-modified electrode samples (scattered points), each with the linear fitting (solid lines). Area of working electrode, linear equations with coefficient values, and sensitivity values with standard deviations of each sample are additionally listed in the tables. The error bars represent the standard deviation from three times repeated measurements.

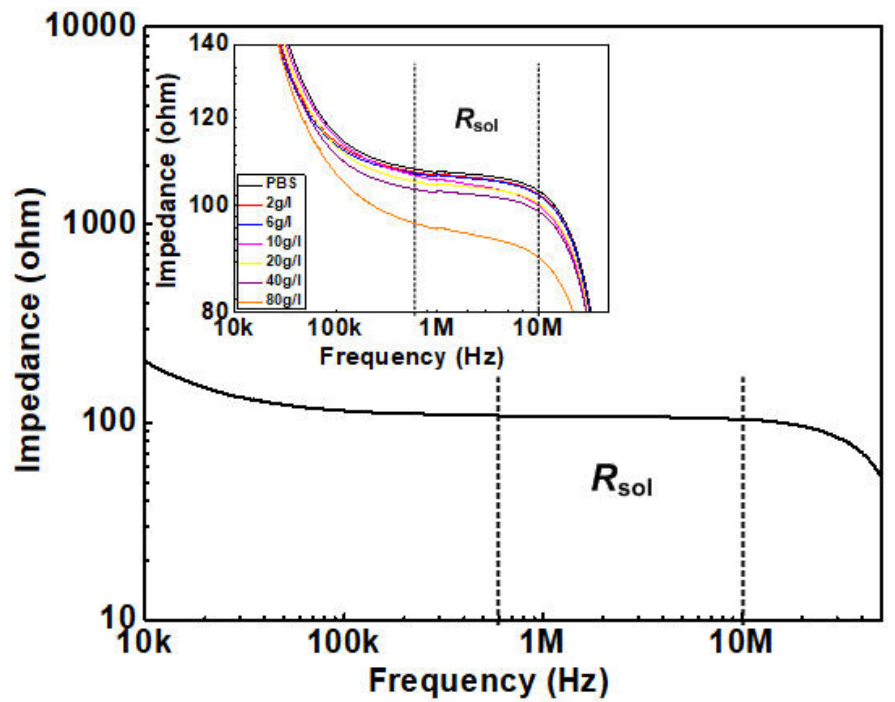

Fig. 5. Impedance curve of IDEs cell density sensor in $10 \mathrm{mM}$ PBS solution. The inset shows the impedance curves of IDEs cell mass sensor in PBS solutions and various concentrations of yeast suspensions $(2-80 \mathrm{~g} / \mathrm{l})$.
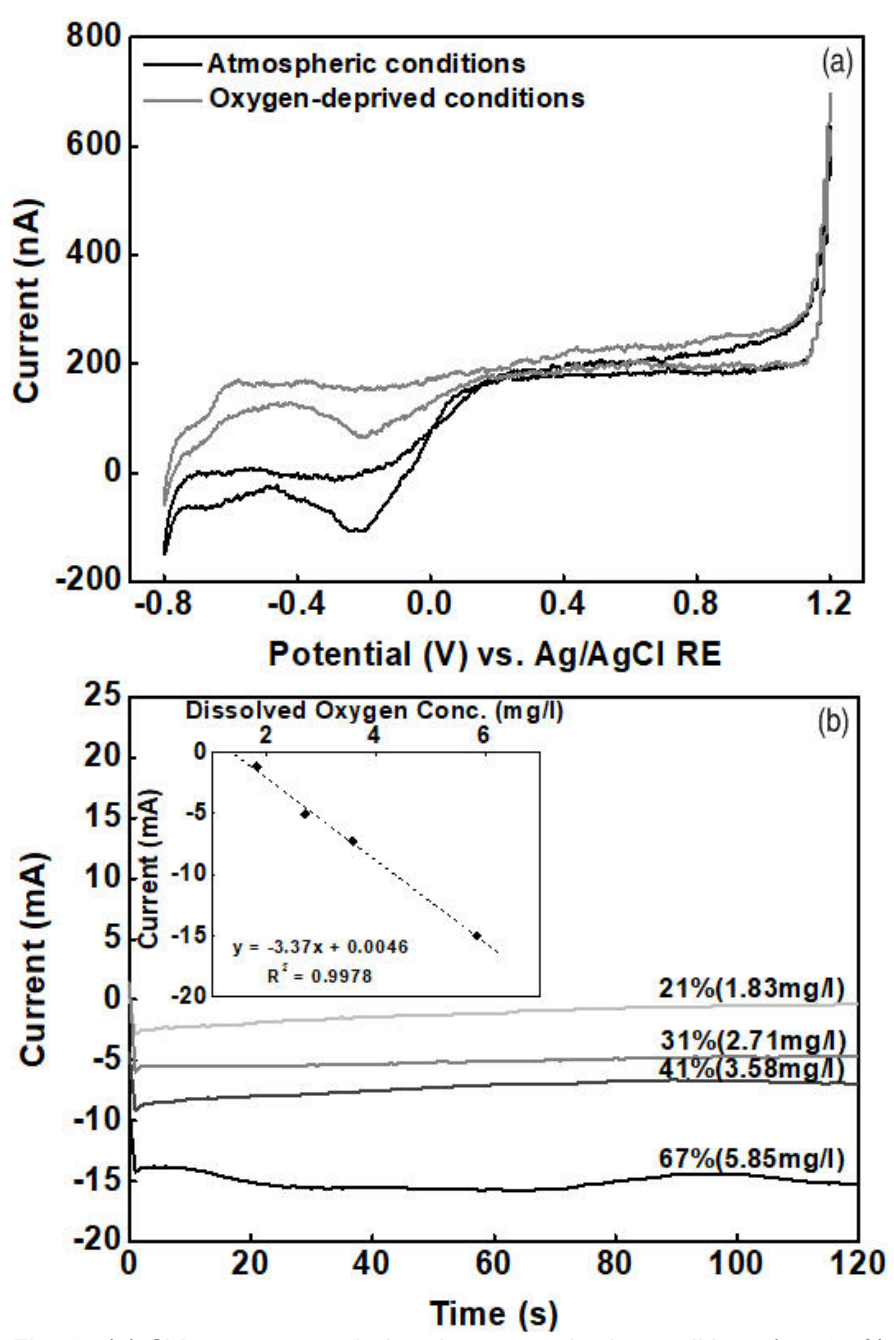

Fig. 6. (a) CV curves recorded under atmospheric conditions (ca. $67 \%$ oxygen) and in an oxygen-deprived conditions (ca. $25 \%$ ) using $10 \mathrm{mM}$ PBS at pH 7.4 with a voltage range $-0.8-1.2 \mathrm{~V}$ applied against an $\mathrm{Ag} / \mathrm{AgCl}$ external RE at a scan rate of $10 \mathrm{mV} / \mathrm{s}$, and (b) CA curves of PBS with different oxygen concentration. The inset shows the variations of averaged current as a function of $\mathrm{dO}$ concentrations. 

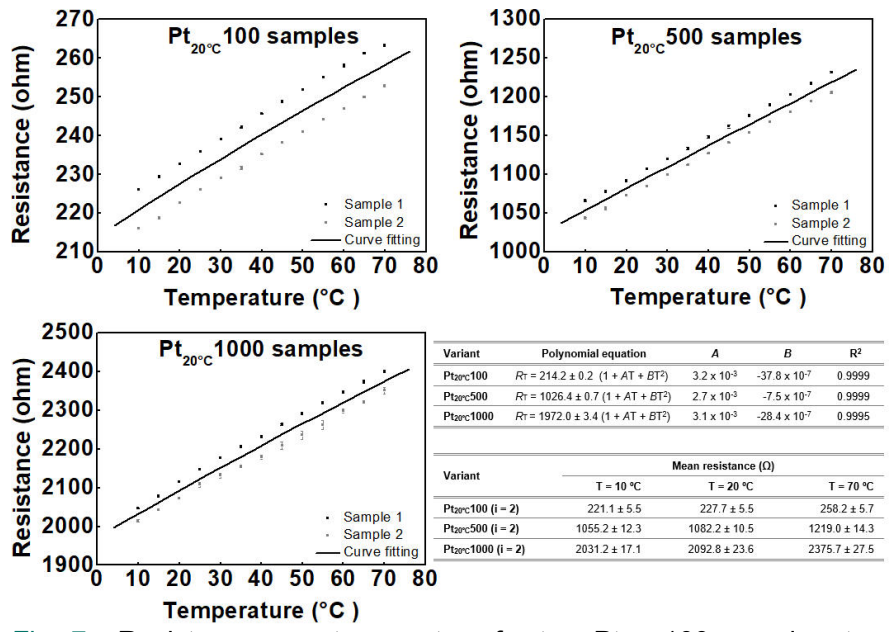

Fig. 7. Resistance over temperature for two $\mathrm{Pt}_{20}{ }^{\circ} \mathrm{C} 100$ samples, two $\mathrm{Pt}_{20}{ }^{\circ} \mathrm{C} 500$ samples, and two $\mathrm{Pt}_{20}{ }^{\circ} \mathrm{c} 1000$ samples (scattered points), each with the polynomial curve fitting (solid lines). Polynomial equations with coefficients values for each sample, and mean values with standard deviations of different samples at $10^{\circ} \mathrm{C}, 20^{\circ} \mathrm{C}$, and $70^{\circ} \mathrm{C}$ are additionally listed in the tables. The error bars represent the standard deviation.

the RTDs leads to an average value of $2.47 \pm 0.35 \times 10^{-7} \Omega \mathrm{m}$, which is also ca. 2-fold higher than the ideal $\rho_{20}{ }^{\circ} \mathrm{C}$ that was used in the calculation. The higher deviation value could be explained by the non-idealities in fabrication process such as the thickness uniformity in thin films. It is worth noting that the calculated average $\alpha$ was comparable to each other with an average value of $0.27 \pm 0.01 \% /{ }^{\circ} \mathrm{C}$. The calculated TCR values were considerably lower than the TCR of pure $\mathrm{Pt}$, which is $0.39 \% /{ }^{\circ} \mathrm{C}$. This was anticipated as the application of adhesion layer between the substrate and the Pt layer is known to further reduce the TCR value [6]. From the results obtained, it is evident that the integrated RTD has the capability for temperature monitoring in bioprocesses.

\section{CONCLUSIONS}

A proof-of-concept of multi-sensor integration of various bio-, chemical and physical sensors on a single chip for monitoring of important parameters in biotechnological processes has been demonstrated. Multiple sensors were fabricated on a single chip using a wafer-level microfabrication process. The integrated enzyme sensors for glucose and lactate showed a linear working range up to a concentration of $10 \mathrm{mM}$. The selectivity and stability of the enzyme sensors responses were also investigated; but the selectivity of lactate sensor was insufficient and require further optimization. The developed glucose sensors had acceptable storage stability. Integrated $\mathrm{pH}$ sensors were governed by a near-Nernstian equation with sensitivity of $60.8 \pm 2.4 \mathrm{mV} / \mathrm{pH}$ with a high regression coefficient. Impedance curves for IDEs cell density sensor revealed an ohmic plateau from approximately $150 \mathrm{kHz}$ to $10 \mathrm{MHz}$ which provided a suitable frequency range for cell density measurements. The absolute averaged current detected in oxygen deprived PBS of dO sensor increases as the dO concentration increases, with good linear correlation of $\mathrm{R}^{2}=0.9978$. Consequently, these findings validated the ability of the MEA-based dO sensor to measure oxygen level in solution. The RTDs showed linear correlation between resistance and temperature, exhibiting temperature dependence of electrical resistance. All sensor elements in the fabricated multi-sensor chip proved their functionality with sufficient sensitivity.

Despite the encouraging results, further studies of the parallel application and reliability issues of all sensors such as stability, repeatability, and reproducibility as well as sterilization of the enzyme-based sensors are yet to be conducted. We envision that the multi-sensor chip eventually will be used for the real-time evaluation, parallel monitoring of glucose, lactate, $\mathrm{pH}$, cell density, dO, and temperature in bioreactors. These systems might not only able to perform highly localized, real-time, multi-parametric electrochemical measurements with different techniques but also to autonomously and continuously monitor and analyze a production vessel, potentially by employing wireless data transmission.

\section{REFERENCES}

[1] S. Mross, T. Zimmermann, N. Winkin, M. Kraft, and H. Vogt, "Integrated multi-sensor system for parallel in-situ monitoring of cell nutrients, metabolites and cell mass in biotechnological processes," Procedia Eng., vol. 120, pp. 372-375, 2015.

[2] M. Pohlscheidt, S. Charaniya, C. Bork, M. Jenzsch, T. L. Noetzel, and A. Luebbert, "Bioprocess and fermentation monitoring," in Encyclopedia of Industrial Biotechnology: Bioprocess, Bioseparation, and Cell Technology, M. C. Flickinger, Ed. John Wiley \& Sons, Inc., 2013, pp. 119.

[3] P. O’Mara, A. Farrell, J. Bones, and K. Twomey, "Staying alive! Sensors used for monitoring cell health in bioreactors," Talanta, vol. 176, pp. 130139, 2018.

[4] M. A. Hanson, X. Ge, Y. Kostov, K. A. Brorson, A. R. Moreira, and G. Rao, "Comparisons of optical $\mathrm{pH}$ and dissolved oxygen sensors with traditional electrochemical probes during mammalian cell culture," Biotechnol. Bioeng., vol. 97, no. 4, pp. 833-841, 2007.

[5] N. D. Lourenço, J. A. Lopes, C. F. Almeida, M. C. Sarraguça, and H. M. Pinheiro, "Bioreactor monitoring with spectroscopy and chemometrics: A review," Anal. Bioanal. Chem., vol. 404, pp. 1211-1237, 2012.

[6] E. E. Krommenhoek et al., "Integrated electrochemical sensor array for on-line monitoring of yeast fermentations," Anal. Chem., vol. 79, no. 15, pp. 5567-5573, 2007.

[7] M. Bäcker et al., "A silicon-based multi-sensor chip for monitoring of fermentation processes," Phys. Status Solidi A, vol. 208, no. 6, pp. 1364 1369, 2011.

[8] T. Geisler, J. Ressler, H. Harz, B. Wolf, and R. Uhl, "Automated multiparametric platform for high-content and high-throughput analytical screening on living cells," IEEE Trans. Autom. Sci. Eng., vol. 3, no. 2, pp. 169-176, Apr. 2006.

[9] X. Guimerà et al., "A minimally invasive microsensor specially designed for simultaneous dissolved oxygen and $\mathrm{pH}$ biofilm profiling," Sensors, vol. 19, pp. 1-13, 2019.

[10] J. Kiefer, N. Ebel, E. Schlücker, and A. Leipertz, "Characterization of Escherichia coli suspensions using UV/Vis/NIR absorption spectroscopy," Anal. Methods, vol. 2, pp. 123-128, 2010.

[11] V. A. Corro-Herrera, J. Gómez-Rodríguez, P. M. Hayward-Jones, D. M. Barradas-Dermitz, M. G. Aguilar-Uscanga, and A. C. Gschaedler-Mathis, "In-situ monitoring of Saccharomyces Cerevisiae ITV01 bioethanol process using near-infrared spectroscopy NIRS and chemometrics," Biotechnol. Prog., vol. 32, no. 2, pp. 510-517, 2016.

[12] E. Tamburini, M. G. Marchetti, and P. Pedrini, "Monitoring key parameters in bioprocesses using near-infrared technology," Sensors, vol. 14, pp. 18941-18959, 2014.

[13] J. Schenk, I. W. Marison, and U. Von Stockar, "pH prediction and control in bioprocesses using mid-infrared spectroscopy," Biotechnol. Bioeng., vol. 100 , no. 1, pp. 82-93, 2008.

[14] B. N. Berry, T. M. Dobrowsky, R. C. Timson, R. Kshirsagar, T. Ryll, and K. Wiltberger, "Quick generation of Raman spectroscopy based inprocess glucose control to influence biopharmaceutical protein product quality during mammalian cell culture," Biotechnol. Prog., vol. 32, no. 1, pp. 224-234, 2016. 
[15] J. Claßen, F. Aupert, K. F. Reardon, D. Solle, and T. Scheper, "Spectroscopic sensors for in-line bioprocess monitoring in research and pharmaceutical industrial application," Anal. Bioanal. Chem., vol. 409, pp. 651-666, 2017.

[16] M. Swan, "Sensor mania! The internet of things, wearable computing, objective metrics, and the quantified self 2.0," J. Sens. Actuator Networks, vol. 1 , no. 3, pp. 217-253, Nov. 2012

[17] K. Mitsubayashi, O. Niwa, and Y. Ueno, Eds., Chemical, Gas, and Biosensors for Internet of Things and Related Applications. Elsevier Inc., 2019.

[18] K. Mitsubayashi, O. Niwa, and Y. Ueno, Eds., Chemical, Gas, and Biosensors for Internet of Things and Related Applications. Elsevier Inc., 2019. [Online]. Available: https://doi.org/10.1016/C2017-0-03327-X.

[19] E. E. Krommenhoek et al., "Lab-scale fermentation tests of microchip with integrated electrochemical sensors for $\mathrm{pH}$, temperature, dissolved oxygen and viable biomass concentration," Biotechnol. Bioeng., vol. 99, no. 4, pp. 884-892, 2008.

[20] N. Todtenberg et al., "Autonomous sensor capsule for usage in bioreactors," IEEE Sens. J., vol. 15, no. 7, pp. 4093-4102, 2015.

[21] A. Weltin, J. Kieninger, and G. A. Urban, "Microfabricated, amperometric, enzyme-based biosensors for in vivo applications," Anal. Bioanal. Chem., vol. 408, no. 17, pp. 4503-4521, Jul. 2016.

[22] S. Mross, T. Zimmermann, S. Zenzes, M. Kraft, and H. Vogt, "Study of enzyme sensors with wide, adjustable measurement ranges for in-situ monitoring of biotechnological processes," Sensors Actuators, B Chem., vol. 241, pp. 48-54, 2017.

[23] S. Mross, "Integrated multi-sensor system for parallel in-situ monitoring of biotechnological processes," Ph.D. dissertation, Dept. Elect. Eng. \& Info. Tech., Universität Duisburg-Essen, Germany, 2016.

[24] I. P. Vincentini, "Design and fabrication of a multi-parameter biochip for bioreactor monitoring," M.S. thesis, Politecnico Di Milano, Italy, 2018.

[25] N. Vanonckelen, "Interfacing digital microfluidics with potentiometric detection techniques," M.S. thesis, Dept. Biosys., KU Leuven, 2016.

[26] A. U. Alam et al., "Polymers and organic materials-based $\mathrm{pH}$ sensors for healthcare applications," Prog. Mater. Sci., vol. 96, no. January, pp. 174 216, 2018.

[27] Y. Qin, H. Kwon, M. M. R. Howlader, and M. J. Deen, "Microfabricated electrochemical $\mathrm{pH}$ and free chlorine sensors for water quality monitoring: recent advances and research challenges," RSC Adv., vol. 5, pp. 69086-69109, 2015.

[28] O. Korostynska, K. Arshak, E. Gill, and A. Arshak, "Review on state-ofthe-art in polymer based pH sensors," Sensors, vol. 7, no. 12, pp. 30273042, 2007.

[29] M. T. Ghoneim et al., "Recent progress in electrochemical ph-sensing materials and configurations for biomedical applications," Chem. Rev., vol. 119, pp. 5248-5297, 2019.

[30] G. Fomo, T. T. Waryo, P. Baker, and E. I. Iwuoha, "Electrochemical deposition and properties of polyaniline films on carbon and precious metal surfaces in perchloric acid/ acetonitrile," Int. J. Electrochem. Sci, vol. 11, pp. 10347-10361, 2016.

[31] J. P. Carvell and J. E. Dowd, "On-line measurements and control of viable cell density in cell culture manufacturing processes using radio-frequency impedance," Cytotechnology, vol. 50, pp. 35-48, 2006.

[32] K. Asami and T. Yonezawa, "Dielectric analysis of yeast cell growth," Biochim. Biophys. Acta, vol. 1245, pp. 99-105, 1995.

[33] L. Yang, "Electrical impedance spectroscopy for detection of bacterial cells in suspensions using interdigitated microelectrodes," Talanta, vol. 74, no. 5, pp. 1621-1629, 2008.

[34] F. van Rossem et al., "Sensing oxygen at the millisecond time-scale using an ultra-microelectrode array (UMEA)," Sensors Actuators B, vol. 238, pp. 1008-1016, 2017.

[35] W. Y. Du, Resistive, Capacitive, Inductive, and Magnetic Sensor Technologies. CRC Press, 2015.

[36] R. Murji and M. J. Deen, "A Scalable Meander-Line Resistor Model for Silicon RFICs," IEEE Trans. Electron Devices, vol. 49, no. 1, pp. 187190, 2002.

[37] R. Murji and M. J. Deen, "A scalable meander-line resistor model for silicon RFICs," IEEE Trans. Electron Devices, vol. 49, no. 1, pp. 187190, 2002.

[38] P. K. H. Ronkainen, H. Kattelus, E. Tarvainen, T. Ruhisaari, M. Andersson, "IC compatible planar inductors on silicon," in IEE Proceedings - Circuits, Devices and Systems, 1997, pp. 29-35.

[39] E. Fiedler, M. F. Porto Cruz, O. F. Cota Monjarás, and T. Stieglitz, "Evaluation of thin-film temperature sensors for integration in neural probes," in International IEEE/EMBS Conference on Neural
Engineering, NER, Jul. 2015, vol. 2015-July, pp. 549-552.

[40] A. Brandenburg, J. Kita, A. Groß, and R. Moos, "Novel tube-type LTCC transducers with buried heaters and inner interdigitated electrodes as a platform for gas sensing at various high temperatures," Sensors Actuators $B$, vol. 189 , pp. 80-88, 2013.

[41] A. Hassibi, R. Navid, R. W. Dutton, and T. H. Lee, "Comprehensive study of noise processes in electrode electrolyte interfaces," J. Appl. Phys., vol. 96, p. 69903, 2004.

[42] M. Lambrechts and W. Sansen, Biosensors: Microelectrochemical Devices, 1st ed. Great Britain: Taylor \& Francis Group, 1992.

[43] Y. Liu, X. Luo, W. Shi, and Y. Cui, "Microplate-integrated biosensors for glucose and lactate," J. Electrochem. Soc., vol. 166, no. 6, pp. B421B425, Apr. 2019.

[44] T. B. Goriushkina, A. P. Soldatkin, and S. V. Dzyadevych, "Application of amperometric biosensors for analysis of ethanol, glucose, and lactate in wine," J. Agric. Food Chem, vol. 57, pp. 6528-6535, 2009.

[45] N. Sato and H. Okuma, "Amperometric simultaneous sensing system for d-glucose and 1-lactate based on enzyme-modified bilayer electrodes," Anal. Chim. Acta, vol. 565, pp. 250-254, 2006.

[46] A. Weltin, B. Enderle, J. Kieninger, and G. A. Urban, "Multiparametric, flexible microsensor platform for metabolic monitoring in vivo," IEEE Sens. J., vol. 14, no. 10, pp. 3345-3351, 2014.

[47] T. Lindfors and A. Ivaska, "pH sensitivity of polyaniline and its substituted derivatives," J. Electroanal. Chem., vol. 531, pp. 43-52, 2002.

[48] A. A. Karyakin et al., "Processible polyaniline as an advanced potentiometric $\mathrm{pH}$ transducer. Application to biosensors," Anal. Chem., vol. 71, no. 13, pp. 2534-2540, 2002.

[49] B. Lakard, G. Herlem, S. Lakard, R. Guyetant, and B. Fahys, "Potentiometric pH sensors based on electrodeposited polymers," Polymer (Guildf)., vol. 46, no. 26, pp. 12233-12239, 2005.

[50] B. Lakard, G. Herlem, S. Lakard, R. Guyetant, and B. Fahys, "Potentiometric pH sensors based on electrodeposited polymers," Polymer, vol. 46, no. 26, pp. 12233-12239, 2005.

[51] V. S. Turkani et al., "Nickel based RTD fabricated via additive screen printing process for flexible electronics," IEEE Access, vol. 7, pp. 37518$37527,2019$.

[52] S. Dutta et al., "Thickness dependence of the resistivity of platinum-group metal thin films," J. Appl. Phys, vol. 122, p. 25107, 2017.

[53] G. Hegde, M. Povolotskyi, T. Kubis, J. Charles, and G. Klimeck, “An environment-dependent semi-empirical tight binding model suitable for electron transport in bulk metals, metal alloys, metallic interfaces, and metallic nanostructures. II. Application - Effect of quantum confinement and homogeneous strain on Cu conductance," J. Appl. Phys., vol. 115, no. 12, p. 123704, Mar. 2014

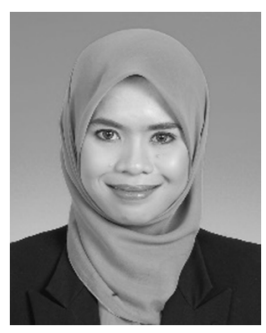

Nurul Izni Rusli was born in Kelantan, Malaysia. She received the B.Eng. in electrical-electronics engineering and M.Eng. in electrical from Universiti Teknologi Malaysia (UTM), Malaysia, in 2008 and 2013, respectively. Since 2013, she has been working at Universiti Malaysia Perlis, Malaysia. She is currently pursuing the Ph.D degree in electrical engineering at $\mathrm{KU}$ Leuven, Belgium under sponsorship Skim Latihan Akademik Bumiputera (SLAB) reference no. KPT(BS)860703295192. Her research interest includes micro- and nanosystems towards bio-chemical sensors applications.

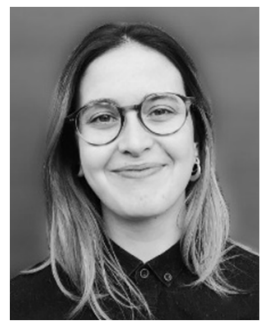

Irene Pia Vincentini was born in Lecce, Italy, in 1994. She attended Politecnico of Milan, Italy to pursue Bachelor degree in Biomedical Engineering and Master degree in Electronics Engineering. In 2018-19, she took part in the Erasmus Internship at ESAT Department, KU Leuven, Belgium under the supervision of Prof. Michael Kraft where she conducted her Master thesis entitled "Design and Fabrication of a MultiParameter Biochip for Bioreactor Monitoring". She is currently working as electronics engineer in Milan, Italy. 


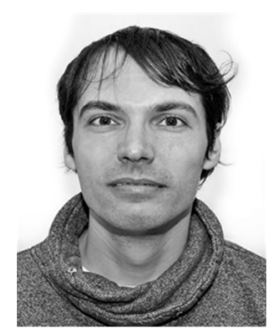

Frederik Ceyssens was born in Sint-Truiden, Belgium, in 1980. He was a research assistant at ESAT-MICAS, where he also obtained his PhD in 2009 under supervision of Prof. Robert Puers. The focus of his Ph.D was microfabrication processes in polymers and application thereof. Afterwards, he started working as a research fellow of the FWO, researching novel braincomputer interfaces in a collaboration between $\mathrm{KU}$ Leuven and the Gathuisberg university hospital. Currently, he is a research expert at the KU Leuven Nanocentre, focusing on micro- and nanofabrication technologies and neural implants for long-term (chronic), high resolution neural stimulation, and recording.

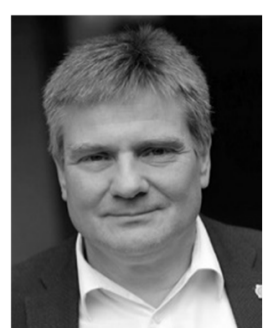

Michael Kraft joined the MICAS group as a full professor of micro- and nanosystems in October 2017. He brings along 20 years' experience in the design, fabrication and characterization of a wide range of micro- and nanosystems and micro-electro-mechanical systems (MEMS) sensors, intelligent interface circuits and control systems for micro-devices, atom and ion chips, bio-medical and biochemical sensors, and devices. These topics will also form the framework of his future research, with a strong focus on bio-medical sensors and systems. He is the lead of the cleanroom and MEMS activities in the Leuven Nanocentre. 